\title{
Atividades de modelagem computacional no auxílio à interpretação de gráficos da Cinemática
}

(Computational modelling activities to help in Kinematics graphs interpretation)

\author{
Ives S. Araujo ${ }^{1}$, Eliane A. Veit e Marco A. Moreira \\ ${ }^{1}$ Instituto de Física, Universidade Federal do Rio Grande do Sul \\ Recebido em 01/12/03; Aceito em 08/04
}

\begin{abstract}
Neste artigo analisamos as principais dificuldades dos estudantes na interpretação de gráficos da Cinemática e apresentamos dois subprodutos diretos de um trabalho de pesquisa voltado para a superação destas dificuldades. Estes subprodutos estão relacionados a um conjunto de atividades de modelagem computacional complementares às atividades tradicionais de ensino da Cinemática e a adaptação e validação de um teste sobre o entendimento de gráficos da cinemática.

Palavras-chave: modelagem computacional, gráficos de Cinemática, ensino de Física.
\end{abstract}

In this paper we analyze the main student difficulties in the interpretation of kinematics graphs and we present two byproducts of our research oriented to overcome these difficulties: a set of complementary teaching activities involving computational modelling and an adaptation of a test about understanding of kinematics graphs to Portuguese with its respective validation.

Keywords: computational modelling; kinematics, physics instruction.

\section{Introdução}

O presente artigo está embasado em um trabalho de pesquisa onde se investigou a possibilidade de propiciar condições favoráveis à aprendizagem significativa (em um referencial ausubeliano; [1]) na interpretação de gráficos da Cinemática através da aplicação de atividades de modelagem computacional, complementares às atividades tradicionais de ensino deste conteúdo. A populaçãoalvo deste estudo foi constituída por calouros do curso de Física da UFRGS no $2^{\circ}$ semestre letivo de 2002 . Resultados de pesquisa em ensino de Física [2, 3] apontam para uma série de dificuldades que os alunos enfrentam na interpretação de gráficos da cinemática. No Brasil, Agrello e Garg [4] traduziram o teste TUG-K (Teste sobre o entendimento de gráficos da Cinemática) e o aplicaram aos alunos da UnB, identificando em nossos estudantes as mesmas dificuldades apontadas por Beichner [3]. A seção 2 está dedicada à apresentação dos resultados dessas pesquisas. Em particular, o resultado mais relevante para o presente trabalho é o da indicação da interatividade entre o estudante e o experimento como o fator decisivo nas situações onde houve aprendizagem. Isto nos levou a considerar a hipótese de que o uso de um software de modelagem computacional pudesse, também, contribuir para a efetivação de uma aprendizagem significativa. Acreditamos que dentre as várias formas em que o computador vêm sendo (sub)utilizado nas práticas de ensino, a modelagem computacional seja a que melhor permita a interação dos estudantes com o processo de construção e análise do conhecimento científico, permitindo que compreendam melhor

\footnotetext{
${ }^{1}$ Enviar correspondência para Ives S. Araujo. E-mail: ives@ if.ufrgs.br.
}

os modelos físicos e discutam o contexto de validade dos mesmos. Optamos pelo software Modellus, cujas características essenciais são apresentadas na seção 3. Nela apresentamos os subprodutos desenvolvidos dentro de nosso trabalho de pesquisa [5]: um conjunto de atividades computacionais que elaboramos utilizando o software Modellus visando à superação das dificuldades de interpretação de gráficos da Cinemática e a adaptação para o português de um teste sobre o entendimento de gráficos em Cinemática (TUG-K; Beichner [3]), ampliado e validado. Os comentários e resultados finais são apresentados na seção 4 .

\section{Interpretação de gráficos da Cinemática}

Uma das habilidades requeridas para a compreensão de conteúdos de Física é a construção e interpretação de gráficos. Em um gráfico uma grande quantidade de informação pode ser resumida. Ser capaz de extrair informações de um gráfico é uma habilidade de cientistas e professores, porém muitas vezes pouco compreendida pelos estudantes. Gráficos da cinemática, i.e., gráficos de posição, velocidade e aceleração em função do tempo são, geralmente, os primeiros trabalhados em um curso de Física. Propiciar condições para que os alunos aprendam a interpretá-los e utilizá-los como uma das possíveis representações de fenômenos físicos contribui, não somente para a aprendizagem da cinemática, mas também

Copyright by the Sociedade Brasileira de Física. Printed in Brazil. 
para a aprendizagem futura de outros conteúdos. Vários estudos encontrados na literatura [6-10] narram o desenvolvimento de propostas de ensino de Física que foram bem sucedidas em aumentar as habilidades de interpretação de gráficos a partir de experiências de aquisição de dados em tempo real utilizando o computador (propostas MBL: "Microcomputer-Based Laboratory"). Os pesquisadores começaram inicialmente a investigar a que se devia esta melhora na interpretação. Uma das dificuldades que mais apareceram nos trabalhos foi a da interpretação errônea, por parte dos alunos, dos gráficos como fotografias da trajetória do movimento. As melhorias promovidas pelas atividades utilizando MBL pareciam estar intimamente vinculadas a esta questão, pois estas atividades permitiam aos alunos observar o traçado em tempo real dos gráficos conforme a experiência se desenvolvia. Em algumas experiências [6,7], os alunos usavam o próprio corpo como objeto de estudo na análise dos movimentos. Esses movimentos eram detectados através de sensores e os dados obtidos eram utilizados para traçar gráficos cinemáticos na tela do computador. Suspeita-se que esta interatividade seja a força motriz da melhoria na interpretação de gráficos ocasionada pelas atividades envolvendo MBL. Beichner [9] propôs um estudo onde o feedback sinestésico fosse completamente removido, somente dando aos estudantes réplicas visuais de situações de movimento. A produção dos gráficos foi sincronizada com a reanimação do movimento de forma que os estudantes pudessem ver o objeto se movendo e o traçado de um gráfico cinemático, correspondente a este movimento, simultaneamente. Os resultados obtidos neste estudo indicam que esta técnica não apresentou uma vantagem educacional sobre a forma de instrução tradicional. O autor argumenta que desde que Brassel [6] e outros pesquisadores demonstraram a superioridade das práticas utilizando MBL em relação às técnicas tradicionais de ensino, os resultados de seu estudo sugerem que a justaposição visual não é uma variável relevante na melhora do desempenho dos alunos na interpretação de gráficos da Cinemática. O fator que realmente faz a diferença segundo Beichner é a interatividade do estudante com o experimento. Entendemos que o uso de uma ferramenta que pudesse descrever os processos dinamicamente, permitindo ao aluno interagir com o movimento dos corpos envolvidos ao mesmo tempo em que observa os gráficos sendo traçados poderia vir a facilitar a sua compreensão do evento. Outro aspecto importante a ser salientado é o da motivação para aprender proporcionada por este tipo de interação. Além do interesse natural despertado pelo uso de microcomputadores, os resultados obtidos por Araujo [5] sugerem que a aplicação de atividades de modelagem, como as descritas neste artigo, exerce uma influência positiva na predisposição do indivíduo para aprender Física. Isto ocorre na medida em que a relevância de determinadas relações matemáticas e conceitos é percebida pelo aluno durante o processo de interação com os modelos conceituais, permitindo que o conteúdo visto anteriormente por ele, e que até então estava muito abstrato, passe a ter um referencial mais concreto. Segundo Beichner [10], a crença de que os gráficos são uma espécie de fotografia do movimento é, provavelmente, a principal confusão que os alunos fazem ao se depararem com gráficos da Cinemática. Como um exemplo óbvio desta situação, imaginemos um garoto numa bicicleta descendo uma colina e depois ficando sobre um pequeno morro. Quando os alunos são solicitados a traçarem gráficos cinemáticos relevantes da situação, freqüentemente o que é traçado é um gráfico y vs. $\mathrm{x}$, mostrando a descida da colina e a subida no pequeno morro ao invés de um gráfico de y (ou qualquer outra variável cinemática) vs. t. Este erro é especialmente problemático quando o movimento horizontal é uma função linear do tempo. No movimento de projéteis, por exemplo, as curvas nos gráficos altura $v s$. alcance e altura vs. tempo têm o mesmo formato parabólico, fazendo com que a visão de gráficos como fotografias do movimento seja difícil de detectar, pois o estudante pode estar trabalhando com um tipo de gráfico, mas pensando em outro. No estudo do tema interpretação de gráficos da Cinemática, McDermott, Rosenquist e Van Zee [2] analisaram as narrativas feitas pelos estudantes durante o processo de elaboração e análise dos gráficos e identificaram 10 das principais dificuldades apresentadas por esses alunos ao trabalharem com gráficos cinemáticos. Cinco destas dificuldades estão em conectar os gráficos aos conceitos físicos: a) discriminar entre inclinação e altura; b) interpretar mudanças na altura e mudanças na inclinação; c) relacionar um tipo de gráfico a outro; d) relacionar a narração de um movimento com um gráfico que o descreve; e) interpretar a área sob o gráfico. As outras cinco dificuldades encontradas estão em conectar gráficos ao mundo real: a) representar movimento contínuo por uma linha contínua; b) separar a forma de um gráfico da trajetória do movimento; c) representar velocidade negativa; d) representar aceleração constante; e) fazer distinção entre diferentes tipos de gráficos do movimento. Tendo em vista os resultados deste trabalho Beichner [3] propôs o desenvolvimento e a análise de um teste para averiguar a interpretação de gráficos da Cinemática por parte dos estudantes, fazendo também o levantamento de suas principais dificuldades. Ele argumenta que os professores de Física utilizam gráficos como uma segunda linguagem de comunicação, admitindo que seus estudantes possam obter uma descrição detalhada do sistema físico analisado, através deste tipo de representação. Infelizmente, seu trabalho indica que os estudantes não compartilham do mesmo vocabulário que os professores. Todos os estudantes que participaram do teste já haviam sido expostos à Cinemática. A Tabela 1 descreve quais foram os objetivos abordados pelo teste e a Tabela 2 refere-se às dificuldades que foram mapeadas através da utilização do teste.

Tabela 1 - Objetivos do teste TUG-K de compreensão de gráficos da Cinemática (Adaptado de Beichner [2]).

\begin{tabular}{|c|c|}
\hline Dado & O Estudante deverá \\
\hline $\begin{array}{l}\text { 1) Gráfico de posição vs. } \\
\text { tempo }\end{array}$ & Determinar a velocidade \\
\hline $\begin{array}{l}\text { 2) Gráfico da velocidade } v s \text {. } \\
\text { tempo }\end{array}$ & Determinar a aceleração \\
\hline $\begin{array}{l}\text { 3) Gráfico da velocidade } v s \text {. } \\
\text { tempo }\end{array}$ & Determinar o deslocamento \\
\hline $\begin{array}{l}\text { 4) Gráfico da aceleração vs. } \\
\text { tempo }\end{array}$ & $\begin{array}{l}\text { Determinar a variação } \\
\text { velocidade }\end{array}$ \\
\hline 5) Gráfico da Cinemática & $\begin{array}{l}\text { Selecionar outro gráfico } \\
\text { correspondente }\end{array}$ \\
\hline 6) Gráfico da Cinemática & $\begin{array}{l}\text { Selecionar a descrição textual } \\
\text { adequada }\end{array}$ \\
\hline $\begin{array}{l}\text { 7) Descrição textual do } \\
\text { movimento }\end{array}$ & $\begin{array}{l}\text { Selecionar } \\
\text { correspondente }\end{array}$ \\
\hline
\end{tabular}


Tabela 2 - Dificuldades dos estudantes em interpretação de gráficos da Cinemática (Adaptado de Beichner [2]).

\section{Dificuldades}

1) Visão de gráficos como uma fotografia do movimento

2) Confusão entre altura e inclinação

3) Confusão entre variáveis cinemáticas

4) Erros quanto à determinação de inclinações de linhas que não passam pela origem

5) Desconhecimento do significado das áreas no gráfico abaixo das curvas cinemáticas

6) Confusão entre área/inclinação/altura

\section{A ferramenta de modelagem com- putacional Modellus}

Dentre as ferramentas de modelagem disponíveis atualmente, o Modellus [11] destaca-se por permitir que estudantes e professores façam experimentos conceituais utilizando modelos matemáticos definidos a partir de funções, derivadas, taxas de variação, equações diferenciais e equações a diferenças finitas, escritos de forma direta, ou seja, assim como o aluno aprendeu na sala de aula sem a necessidade de metáforas simbólicas, tais como os diagramas de Forrester utilizados nos modelos confeccionados com o STELLA [12]. Outra característica importante provida pelo Modellus é a representação múltipla, i.e., o usuário pode criar, ver e interagir com as representações analíticas, analógicas e gráficas dos objetos matemáticos [13]. O Modellus possui uma interface gráfica intuitiva, o que vem a facilitar a interação dos estudantes com modelos em tempo real e a análise de múltiplas representações desses modelos, permitindo também, observar múltiplos experimentos (conceituais) simultaneamente. Vale a pena destacar que o Modellus é um programa de distribuição gratuita e vem sendo muito utilizado em diversos países, tendo sido traduzido para vários idiomas (inglês, espanhol, eslovaco, grego e português do Brasil). Buscando evidenciar as potencialidades do Modellus, Teodoro [13] o analisa através de dois pontos de vista. Do ponto de vista computacional, o programa pode ser visto como um micromundo no computador para uso tanto dos estudantes quanto dos professores, não sendo baseado numa metáfora de programação. Na "janela do modelo" o usuário pode escrever modelos matemáticos, quase sempre da mesma forma que a manuscrita do dia-a-dia, dispensando o aprendizado de uma nova linguagem para a elaboração desses modelos. Do ponto de vista educacional, o Modellus incorpora tanto o modo expressivo quanto o modo exploratório das atividades de aprendizagem (Bliss e Ogborn apud Teodoro [13]). Em uma atividade de aprendizagem expressiva, os estudantes podem construir seus próprios modelos matemáticos e criar diversas formas para representá-los. No modo exploratório, os alunos podem usar modelos e representações feitos por outros, analisando como grandezas diferentes se relacionam entre si ou visualizando a simulação de um evento físico. O delineamento pedagógico do Modellus admite que o computador é uma ferramenta cognitiva, mas não substitui habilidades humanas de alta ordem, ou seja, admite-se que o Modellus auxilia na aprendizagem, mas que a "inteligência, emoção, cultura, poesia e arte residem no usuário, não no software" [14]. Trabalharemos aqui com a idéia de modelos físicos vistos como descrições simplificadas e idealizadas de sistemas ou fenômenos físicos, aceitos pela comunidade científica, que envolvem elementos como representações (externas), proposições semânticas e modelos matemáticos subjacentes. Entenderemos modelagem como um processo de criação de um modelo, dividido em cinco estágios não-hierárquicos: seleção, construção, validação, análise e expansão do modelo, "onde os três estágios intermediários sobrepõem-se, podendo ser conduzidos ao mesmo tempo" [15].

\section{Atividades de modelagem com- putacional e teste para avaliação dos conhecimentos}

Tencionamos neste trabalho colocar à disposição da comunidade um conjunto de atividades complementares de ensino de Física envolvendo a exploração e a construção de modelos computacionais como um processo de interação (experimento-aluno) desenvolvidas em nosso trabalho de pesquisa [5]. A exploração desse tipo de modelo faz com que o estudante se questione constantemente sobre os efeitos de suas ações sobre os resultados gerados pelo modelo computacional, normalmente esta questão pode ser descrita como: - se eu alterar "isso" o que acontece com "aquilo"? Este raciocínio causal subjacente servirá como pano de fundo para a promoção da interatividade. As atividades de ensino foram elaboradas com o intuito de levar o aluno a questionar-se sobre as relações existentes, entre os conceitos cinemáticos e os gráficos do movimento de um determinado móvel em questão. Apresentamos aqui alguns exemplos das atividades desenvolvidas. O conjunto completo de 12 atividades exploratórias e 11 expressivas está disponível em:

$$
\begin{gathered}
\text { http://www.if.ufrgs.br/gpef/graficos_ } \\
\text { cinematica.zip }
\end{gathered}
$$

Estas atividades foram elaboradas com o Modellus para auxiliar os alunos na superação de eventuais dificuldades enfrentadas por eles na interpretação de gráficos da Cinemática, apresentadas na Tabela 2. Como já foi destacado anteriormente, um dos princípios norteadores na elaboração das atividades foi o de que estas teriam um caráter complementar às aulas tradicionais, e não a finalidade de substituí-las. Em nosso trabalho de pesquisa [5] verificamos uma melhoria significativa no desempenho dos estudantes que participaram de quatro encontros de $2 \mathrm{~h} 15 \mathrm{~min}$ cada, trabalhando em duplas ou individualmente [17] em um laboratório de computação. A melhoria no desempenho dos estudantes foi medida utilizando uma adaptação para a língua portuguesa do TUG$\mathrm{K}$ (Teste sobre o entendimento de gráficos da Cinemática), ao qual acrescentamos quatro questões extras às 21 questões de escolha simples com cinco alternativas cada, proposto por Beichner [3]. Essas questões adicionais foram elaboradas tendo como base os problemas propostos por McDermott, Rosenquist e Van Zee [2] e seguindo os mesmos objetivos descritos na Tabela 1. A versão adaptada do teste foi examinada por uma banca de seis doutores em Física da UFRGS especialistas no conteúdo e depois aplicada a uma turma piloto com a intenção de obter o coeficiente de fidedignidade [18] do instrumento (alfa de Cronbach) que foi igual a 0,84. Não adotamos a versão traduzida do teste para o português feita por 
Agrello e Garg [4] porque não há qualquer referência à validação do teste traduzido e, também, porque em algumas questões, mesmo na versão original, o enunciado não é suficientemente rigoroso. A nossa versão validada do TUG-K encontra-se disponível em:

$$
\text { http://www.if.ufrgs.br/gpef/tugk.zip }
$$

Em relação às atividades propostas, cada uma delas foi construída a partir das seguintes considerações (explícitas para o caso das atividades exploratórias e implícitas nas atividades expressivas):

- objetivos a serem alcançados (Tabela 1);

- dificuldades a serem trabalhadas (Tabela 2);

- descrição geral do modelo;

enunciados das atividades que os alunos receberam na forma impressa (estes mesmos enunciados também estão na janela Notas em cada modelo). Como exemplo de atividade exploratória apresentamos o modelo Noel_bar.mdl explicitando as considerações acima citadas:

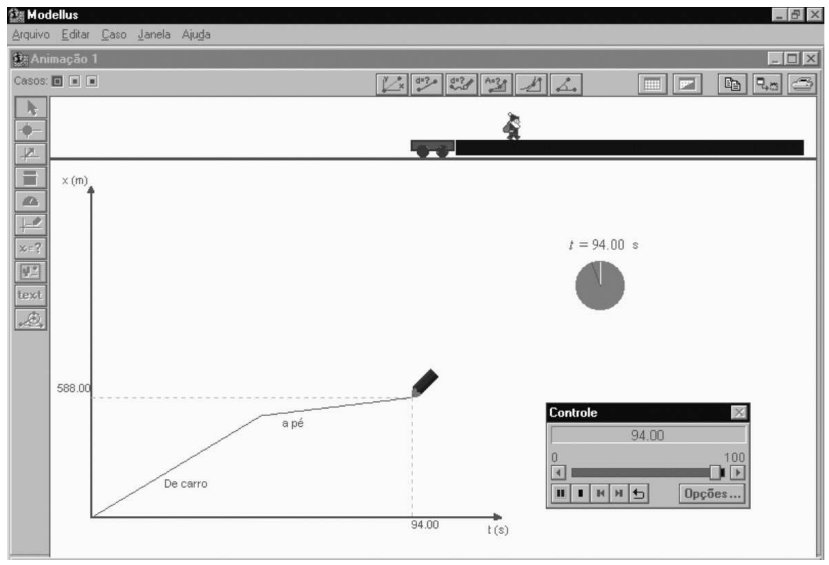

Figura 1 - Tela ilustrativa do modelo Noel_bar.mdl.

\section{a) Objetivos a serem alcançados:}

- Dado o gráfico da posição vs. tempo, o estudante deverá ser capaz de determinar a velocidade.

- Dado um gráfico cinemático qualquer, o estudante deverá ser capaz de descrever textualmente o movimento.

- A partir da descrição do movimento o estudante deverá ser capaz de elaborar o(s) gráfico(s) adequado(s).

\section{b) Dificuldades a serem trabalhadas:}

- Visão de gráficos como uma fotografia do movimento.

- Confusão entre altura e inclinação.

- Confusão entre variáveis cinemáticas.

- Erros quanto à determinação de inclinações de linhas que não passam pela origem.

\section{c) Descrição geral:}

Ao executar este modelo, o Papai Noel está parado sobre o carrinho que se move da esquerda para direita na tela. O carrinho se choca, então, contra a barra azul horizontal e o Papai Noel segue caminhando sobre esta. O gráfico posição contra tempo do Papai
Noel é traçado simultaneamente com o movimento do carrinho. Três casos distintos são propostos: $V a>V b ; V a=V b$ e $V a<V b$, onde $V a$ corresponde à velocidade do Papai Noel de carro e $V b$ à velocidade do Papai Noel a pé.

\section{d) Enunciado:}

1. Execute o modelo e observe com atenção as grandezas e o gráfico. Que tipo de trajetória tem o Papai Noel, quando se move com o carro? E quando está a pé?

2. Qual a distância percorrida pelo Papai Noel 10 segundos após deixar o carro?

3. Qual é o valor da velocidade do Papai Noel, quando ele está no carro? E a pé?

4. Esboce o gráfico de $x$ vs. $t$ para o caso em que o Papai Noel anda a pé e de carro com a mesma velocidade.

5. Esboce o gráfico de $x$ vs. $t$ para o caso em que o Papai Noel anda a pé com velocidade maior do que de carro.

6. Na janela Animação 1 acione os botões verde e rosa (ao lado de "casos:") e compare os gráficos com os seus esboços.

7. É possível, apenas observando o gráfico da posição vs. tempo, determinar em qual trecho o Papai Noel foi mais veloz? Como?

Os exercícios a seguir exemplificam as atividades expressivas em nossa proposta:

1) Construa um modelo que permita obter os seguintes gráficos e animações (em alguns casos é necessário estimar alguns valores...):

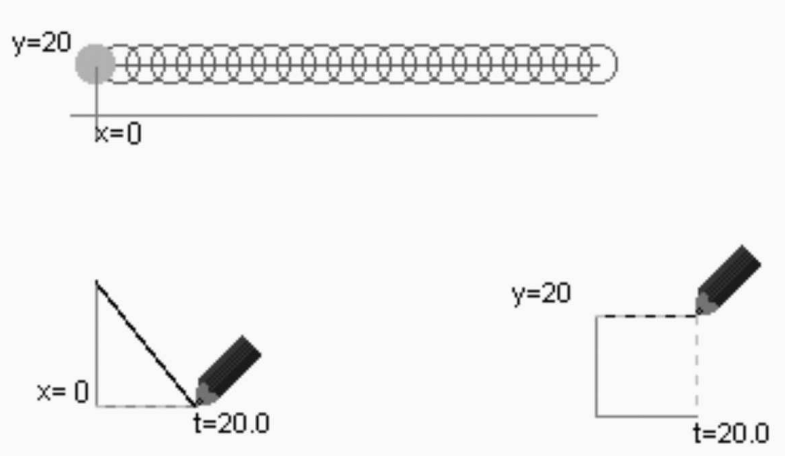

Figura 2 - Gráficos e animações da atividade expressiva 1.

2) Você dispõe das seguintes figuras de canhões e de alvos. Escolha um canhão e um alvo e coloque-os em dois pontos distantes um do outro na janela Animação. Admitindo [19] $g=9.8 \mathrm{~m} / \mathrm{s}^{2}$ faça a animação da bala do canhão (use uma partícula como projétil) até atingir o alvo. As atividades exploratórias caracterizam-se pela observação, análise e interação do sujeito com modelos já construídos, no intuito de permitir ao aluno a percepção e a compreensão das eventuais relações existentes entre a matemática, subjacente ao modelo, e o fenômeno físico em questão. Neste tipo de atividade, o aluno é motivado a interagir com o modelo computacional a fim de responder questões apresentadas em forma de perguntas dirigidas e "desafios". Esta interação é feita através de 
modificações nos valores iniciais e parâmetros do modelo podendo ser utilizados recursos como "barras de rolagem" e "botões" para facilitar as modificações dos mesmos.

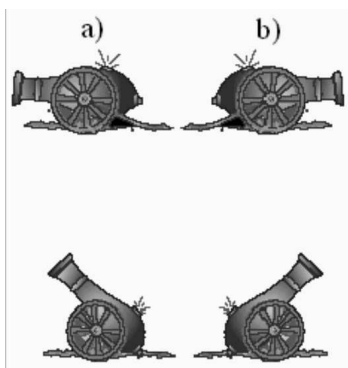

e)

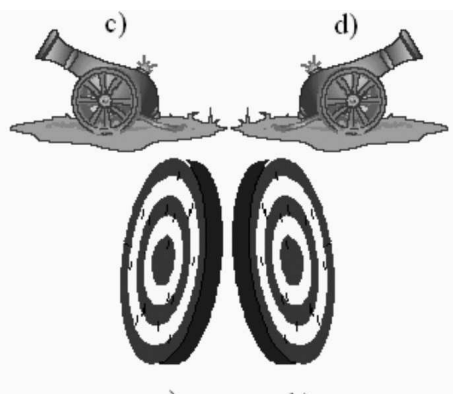

g)

h)
Figura 3 - a) canhão0_e.bmp; b) canhão0_d.bmp; c) canhão30_e.bmp; d) canhão30_d.bmp, e) canhão45_e.bmp; f) canhão45_d.bmp; g) alvo_e.bmp; h) alvo_d.bmp.

As atividades de criação, também conhecidas como atividades expressivas, podem ser caracterizadas pelo processo de construção do modelo desde sua estrutura matemática até a análise dos resultados gerados por ele. Neste tipo de atividade são apresentadas questões que visam à elaboração de modelos a partir de determinados fenômenos de interesse onde podem ser fornecidas tanto informações qualitativas quanto quantitativas do sistema. O aluno pode interagir totalmente com o seu modelo, podendo reconstruí-lo tantas vezes quanto lhe pareça necessário para a produção de resultados que lhe sejam satisfatórios. Cabe aqui ressaltar que em ambos os tipos de atividades a interação entre elas e o aluno, em princípio, deve ser mediada pelo professor tanto em termos de auxílio técnico para a operação do software, como também no esclarecimento de eventuais dúvidas sobre a Física e a Matemática envolvidas no desenvolvimento de seus modelos. Durante a aplicação do material o professor deve estar atento às situações em que o aluno elabora modelos computacionais (ou atribui valores) corretos matematicamente em princípio, e os resultados não são os esperados. Antes de considerar como um mero "erro de ajuste", estas situações podem ser oportunidades ideais para evidenciar o contexto de validade do modelo computacional criado, estando este sempre vinculado aos pressupostos utilizados na teoria subjacente à criação do próprio modelo físico.

\section{Comentários e Conclusões}

Sob a égide do termo "novas tecnologias" muitos recursos, tais como hipermídia e softwares educacionais, vêm sendo utilizados na tentativa de insuflar novos ânimos ao ensino de Física. Apesar do louvável interesse pela inovação e atualização de velhos métodos, muitos relatos do uso destes recursos não vêm acompanhados por uma avaliação criteriosa de suas contribuições ao processo de aprendizagem do aluno; poucos são, ainda, os trabalhos de pesquisa científica nesta área. Aqui apresentamos uma proposta que já foi avaliada nestes termos [20], com o objetivo de divulgar o material produzido [5] de modo que professores da rede escolar possam se apropriar dele. Entendemos que esse tipo de divulgação seja fundamental para diminuir a distância entre o meio acadêmico e a comunidade em geral. Dentre as várias possibilidades de uso da informática no ensino de Física, optamos pela modelagem computacional, por acreditarmos que esta seja a que melhor possibilita a interação dos estudantes com o processo de construção e análise do conhecimento científico, permitindo que compreendam melhor os modelos físicos e discutam o contexto de validade dos mesmos. Dos vários softwares atualmente disponíveis, optamos pelo Modellus por ele ser um software que permite ao aluno fazer experimentos conceituais utilizando modelos matemáticos definidos a partir de funções, derivadas, taxas de variação, equações diferenciais e equações a diferenças finitas, escritos de forma direta, ou seja, assim como o aluno aprendeu na sala de aula. Um outro aspecto positivo em relação ao Modellus é o fato dele ser um software livre sendo distribuído gratuitamente na Internet (http://phoenix.sce.fct.unl.pt/modellus). Este fato, além das potencialidades da ferramenta, contribuiu para que sua utilização ocorresse em nível mundial. Na área de ensino, nossa escolha recaiu sobre o tema interpretação de gráficos da Cinemática, por se tratar de um assunto amplamente discutido na literatura e fundamental na formação de conceitos apresentados posteriormente ao longo dos cursos de Física. Apesar deste artigo abordar especificamente esse tópico, certamente as potencialidades da modelagem computacional podem ser aproveitadas em outros diferentes contextos onde a natureza dinâmica de determinados fenômenos físicos precisem ser explicitadas, possibilitando ao aluno perceber que o estudo destes não se resume a uma mera aplicação de fórmulas. Pesquisas sobre os benefícios advindos do uso de modelagem computacional em outros contextos, seriam, então, necessárias. Para finalizar salientamos novamente a importância de pesquisas científicas que se ocupem em investigar de que forma o aprendiz relaciona e compreende os conceitos físicos trabalhados com o uso do computador e como extrair um proveito máximo deste tipo de ferramenta. Sem pesquisas deste tipo, corremos o risco de prestar um desserviço aos nossos alunos, pois se por um lado estamos empregando métodos e materiais inovadores, por outro lado ignoramos como estes são assimilados por eles, o que pode ocasionar o reforço de pensamentos e atitudes que justamente estamos tentando superar.

\section{Referências}

[1] M.A. Moreira, Aprendizaje Significativo: Teoría e Práctica (Visor, Madrid, 2000), $101 \mathrm{p}$.

[2] L.C. McDermott, M.L. Rosenquist and E.H. van Zee, American Journal of Physics 55, 503 (1987).

[3] R.J. Beichner, American Journal of Physics 62, 750 (1994).

[4] D.A. Agrello and R. Garg, Revista Brasileira de Ensino de Física 21, 103 (1999).

[5] I.S. Araujo, Um Estudo sobre o Desempenho de Alunos de Física Usuários da Ferramenta Computacional Modellus na Interpretação de Gráficos em Cinemática. Dissertação de Mestrado, Instituto de Física, UFRGS, Porto Alegre, 2002.

[6] H. Brassel, Journal of Research in Science Teaching 24, 385 (1987). 
[7] J.R. Mokros and R.F. Tinker, Journal of Research in Science Teaching 24, 369 (1987).

[8] I. Testa, G. Monroy and E. Sassi, International Journal of Science Education 24, 235 (2002).

[9] R.J. Beichner, Journal of Research in Science Teaching 27, 803 (1990).

[10] R.J. Beichner, American Journal of Physics 64, 1272 (1996).

[11] V.D. Teodoro, J.P. Vieira and F.C. Clérigo, Modellus, Interactive Modelling with Mathematics (Knowledge Revolution, San Diego, 1997).

[12] A.C.K. dos Santos, Y. Cho, I.S. Araujo and G.P. Gonçalves, Caderno Catarinense de Ensino de Física 17, 81 (2000).

[13] V.D. Teodoro, in International CoLos Conference new Network-Based Media in Education (Maribor, Slovenia, 1998) p. 13.

[14] V.D. Teodoro, Modellus: Learning Physics with Mathematical Modelling. Unpublished PhD Thesis, Universidade Nova de Lisboa, Lisboa, 2002.
[15] I. Halloun, Journal of Research in Science Teaching 33, 1019 (1996).

[16] M.A. Moreira and F.L. da Silveira, Instrumentos de Pesquisa em Ensino e Aprendizagem: A Entrevista Clínica e a Validação de Testes de Papel e Lápis (EDIPUCRS, Porto Alegre, 1993), $101 \mathrm{p}$.

[17] Mesmo os alunos que optaram por trabalhar individualmente com o computador interagiram com os seus colegas adjacentes.

[18] Quando se deseja utilizar os escores gerados por um instrumento para comparar grupos em médias, podemos tolerar coeficientes da ordem de 0,7. [16]

[19] Aqui separamos a parte inteira da parte decimal do número por um ponto, embora não seja o correto em português, porque esta é a forma adotada no software Modellus.

[20] I.S. Araujo, E.A. Veit and M.A. Moreira, Physics Student's Performance Using Modellus as a Modelling Tool to Improve Kinematics Graphs Interpretation (a ser publicado). 